\title{
Multistability: Uncovering hidden attractors
}

\author{
T. Kapitaniak ${ }^{1, a}$ and G.A. Leonov ${ }^{2, b}$ \\ 1 Division of Dynamics, Lodz University of Technology, 90-924 Lodz, Stefanowskiego 1/15, \\ Poland \\ 2 Faculty of Mathematics and Mechanics, St. Petersburg State University, \\ 198504 Peterhof, St. Petersburg, Russia
}

\begin{abstract}
This topical issue collects contributions exemplifying the recent scientific progress in understanding the dynamics of multistable systems. The individual papers focus on different questions of present day interest in theory and applications of systems with multiple attractors. The particular attention is paid to uncovering and characterizing hidden attractors. Both theoretical and experimental studies are presented.
\end{abstract}

Multistability is a system property which refers to systems that are neither stable nor totally instable, but that alternate between two or more mutually exclusive states (attractors) over time [1-11]. Multistable systems are very sensitive towards noise $[10,11]$, initial conditions $[2,4,6]$ and system parameter [5] so to keep the system on the desired attractor one needs to apply anappropriate controlling scheme [4].

Most of the common examples of both chaotic and regular attractors, like that of van der Pol, Beluosov-Zhabotinsky, Lorenz, Rossler, Chua and many others are located in the neighbouhoods of unstable fixed points (its basins of attraction touch unstable fixed points). Such attractors are called the self-exited attractor and can can be easily localized numerically by the standard computational procedure (one can start with the initial conditions in a small neighborhood of the unstable fixed point on unstable manifold and observe how it is attracted) $[12,13]$.

Recently, it has been shown that multistability is connected with the occurrence of unpredictable attractors [1-11] which have been called the hidden attractors [14-17]. An attractor is called hidden attractor if its basin of attraction does not intersect with small neighborhoods of the unstable fixed point, i.e., the basins of attraction of the hidden atttractors do not contain unstable fixed points and are located far away from such points. For example, the hidden attractor is the periodic or chaotic attractor in the system without equilibria or with the only stable equilibrium (a special case of multistability and coexistence of attractors). Various examples of hidden attractors are presented in [18-25].

Contrary to the self-exited attractors for numerical localization of hidden attractors it is necessary to develop special analytical-numerical procedures, since there are no similar transient processes leading to such attractors from the neighborhoods of the unstable fixed points $[26,27]$.

\footnotetext{
a e-mail: tomaszka@p.lodz.pl

b e-mail: leonov@math.spbu.ru
} 
This special issue on Multistability and Hidden Attractors reviews the current state of art in the research on multistable systems, particularly on hidden attractors and point out the directions of further studies. New examples of hidden attractors, numerical procedures for finding them as well as methods of controlling mustistable systems are presented. Experimental evidence of the existence of hidden attractors is given. Most of the presented cases are stricktly deterministic (ordinary differential equations) but two of them $[32,38]$ deal with nonlinear dynamics assisted by random noise (nonlinear stochastic differential equations).

Paper [28] shows the examples of three-dimensional chaotic systems with different types of equilibrium poins (both hyperbolic and nonhyperbolic). It is shown that for 5 (out of 8) classes of hyperbolic equilibrium points one can expect the existence of hidden attractors. Generally, the results of this paper support the idea that any dynamical behavior not theoretically forbidden (by rigorous theorem) can occur in an appropriately designed system.

The notion of self-excited and hidden attractors for a Lorenz-like system are discussed [29]. It is shown that for cetrain parameters self excited attractors coexist with hidden attractors. The upper estimate of the Lyapunov dimensions of self-ecxited and hidden attractors is derived analytically.

The occurrence of hidden attractors in van der Pol-Duffing oscillator is discussed in [30]. The calculations of the basins of attraction of co-existing attractors and analysis of the births and deaths of hidden attractors are presented.

Several types of new chaotic flows with hidden attractors are reviewed in [31]. The considered flows are divided into three groups: (i) flows with no equilibrium, (ii) flows with equilibrium line, and (iii) flows with a stable equilibrium.

Using a simple bistable perception model, paper [32] demonstrates how the coexisting states can be controlled by periodic modulation. The statistical analysis of transitions between the coexisting attractors is performed.

Multistability and coexistence of different attractors often create unpredictability of the final state. Paper [33] shows how to control multistability in the hidden attractors through the scheme of linear augmentations and achieve monostability. The transition from multiple attractors to the single attractor is confirmed by the calculatiion of the size of the basin of attraction.

The properties of the symmetrical chaotic systems are described in [34]. The authors have shown that such systems provide the possibility of multistability that can be controlled by the independent amplitude control parameter. Through the design of symmetric Rossler systems, a symmetric pair of coexisting chaotic attractors is obtained.

The hidden attractors in the system with an exponential term are studied in [35]. It has been shown that four-dimensional autonomous flow can generate hidden chaotic attractors. A digital hardware platform-based emulator is designed and implemented to verify the numerical results.

The dynamics of an impulsive Goodwin oscilator with time delay is considered in [36]. The paper focuses on the complexity that arises when the time delay exceeds the typical bursting interval of the feedback. It has been shown that under these conditions the system is capable of displaying quasiperiodicity as well as multistability and hidden attractors.

Paper [37] discusses the occurence of various synchronous states in a ring of unidirectionally coupled modified Rossler oscillators with infinite number of solutions (depending on the initial conditions). It has been shown that with the increase of the coupling the infinitely many synchronous states are observed. The calculations have been performed for three and four oscillators but the possible scenarios for larger networks are discussed. 
The peculiarities of attractors in systems with an equilibrium line are studied in [38]. The oscillator with Chua's memristor as well as for the oscillator with a more realistic model of the memristive element are considered. Both numerical simulations and analog experiments are performed for one of the circuits of the memristor oscillator. In particular the problem, how a system with an equilibrium line can be realized in physical experiments and whether the oscillations in such systems are self-sustained, is considered.

The effects of memristor on chaotic dynamical systems are theoretically and experimentally analyzed in [39]. It has been shown that generally the memristor system has less fixed points than the original one.

The qualitative properties of five-dimensional Rikitake model as discussed in [40]. The existence of five-dimensional hyperchaotic hidden attractor has been proven in numerical simulations and analog experiments.

In [41], the projective synchronization of two hidden hyperchaotic systems with unknown parameters are discussed. Based on Lyapunov stability theory adaptive controllers are constructed to achieve adaptive switched generalized function projective synchronization between two different hyperchaotic systems.

Multistability in the large system of the coupled pendula is investigated in [42]. The occurence of patterns of multi-headed chimera states in which pendula clustered in different heads behave differently (oscillate with different frequencies) and create different types of synchronous states (complete or phase synchronization) is discussed.

The effect of delayed feedbacks on the Sprott B system with hidden attractors is disscussed in [43]. The detailed analysis of Hopf bifurcations is shown.

Paper [44] considers three-dimensional dissipative flow with quadratic nonlinearities in which there is no equilibrium. Various types of coexisting hidden attractors have been identified.

Thus, this special issue provides a wide spectrum of current research on multistable systems (particularly on hidden attractors), and we hope that the related researchers in this field will find it useful. We wish to express our appreciation to the authors of all the papers in this special issue for the excellent contributions as well as many reviewers for their high-quality work on reviewing the manuscripts.

\section{References}

1. U. Feudel, Int. J. Bif. Chaos 18, 1607 (2008)

2. M.D. Shrimali, A. Prasad, R. Ramaswamy, U. Feudel, Int. J. Bif. Chaos 18, 1675 (2008)

3. S. Kraut, U. Feudel, Phys. Rev. E 66, 015207 (2002)

4. A.N. Pisarchik, U. Feudel, Phys. Rep. 540, 167 (2014)

5. A. Chudzik, P. Perlikowski, A. Stefanski, T. Kapitaniak, Int. J. Bif. Chaos, 21, 1907 (2011)

6. S.L.T. de Souza, A.M. Batista, I.L. Caldas, R.L. Viana, T. Kapitaniak Chaos, Solitons Fractals 32, 758 (2007)

7. B. Blazejczyk-Okolewska, T. Kapitaniak Chaos, Solitons Fractals 9, 1439 (1998)

8. B. Blazejczyk-Okolewska, T. Kapitaniak Solitons Fractals 7, 1455 (1996)

9. T. Kapitaniak, Phys. Rev E 47, 1408 (1993)

10. T. Kapitaniak, J. Sound Vibration 102, 440 (1985)

11. A. Silchenko, T. Kapitaniak, V.S. Anishchenko, Phys. Rev. E 59, 1593 (1999)

12. H.E. Nusse, J.A. Yorke, E.J. Kostelich, Dynamics: Numerical Explorations (Springer, New York, 1994)

13. T.S. Parker, L.O. Chua, Numerical Algorithms for Chaotic Systems (Springer, New York, 1989)

14. N.V. Kuznetsov, G.A. Leonov, V.I. Vagaitsev, Analytical numerical method for attractor localization of generalized Chua's system, IFAC Proceedings Volumes (IFACPapersOnline) 4, 29 (2010) 
15. G.A. Leonov, N.V. Kuznetsov, V.I. Vagaitsev, Phys. Lett. A 375, 2230 (2011)

16. G.A. Leonov, N.V. Kuznetsov, V.I. Vagaitsev, Physica D 241, 1482 (2012)

17. G.A. Leonov, N.V. Kuznetsov, Int. J. Bif. Chaos 23, 12330002 (2013)

18. M. Molaie, S. Jafari, J.C. Sprott, S.M.R.H., Int. J. Bif. Chaos 23, 1350188 (2013)

19. S. Jafari, J.C. Sprott, Solitons Fractals 57, 79 (2013)

20. A. Kuznetsov, S. Kuznetsov, E. Mosekilde, N. Stankevich, J. Phys. A: Math. Gen. 48, $125101(2015)$

21. V.-T. Pham, S. Jafari, C. Volos, X. Wang, S.M.R.H. Golpayegani, Int. J. Bif. Chaos 24, $1450146(2014)$

22. V.-T. Pham, C. Volos, S. Jafari, Z. Wei, X. Wang, Int. J. Bif. Chaos 24, 1450073 (2014)

23. Z. Wei, Phys. Lett. A 376, 102 (2011)

24. J.C. Sprott, Phys. Lett. A 378, 1361 (2014)

25. U. Chaudhuri, A. Prasad, Phys. Lett. A 378, 713 (2014)

26. V. Bragin, V. Vagaitsev, N.V. Kuznetsov, G.A. Leonov, J. Comput. Syst. Sci. Inter. 50, $511(2011)$

27. G.A. Leonov, N.V. Kuznetsov, Doklady Math. 84, 475 (2011)

28. J.C. Sprott, Eur. Phys. J. Special Topics 224(8), 1409 (2015)

29. G.A. Leonov, N.V. Kuznetsov, T.N. Mokaev, Eur. Phys. J. Special Topics 224(8), 1421 (2015)

30. S. Brezetskyi, D. Dudkowski, T. Kapitaniak, Eur. Phys. J. Special Topics 224(8), 1459 (2015)

31. S. Jafari, J.C. Sprott, F. Nazarimehr, Eur. Phys. J. Special Topics 224(8), 1469 (2015)

32. A.N. Pisarchik, I.A. Bashkirtseva, L.B. Ryashko, Eur. Phys. J. Special Topics 224(8), 1477 (2015)

33. P.R. Sharma, M.D. Shrimali, A. Prasad, N.V. Kuznetsov, G.A. Leonov, Eur. Phys. J. Special Topics 224(8), 1485 (2015)

34. Ch. Li, W. Hu, J.C. Sprott, X. Wang, Eur. Phys. J. Special Topics 224(8), 1493 (2015)

35. V.-T. Pham, S. Vaidyanathan, C.K. Volos, S. Jafari, Eur. Phys. J. Special Topics 224(8), $1507(2015)$

36. Z.T. Zhusubaliyev, E. Mosekilde, A.N. Churilov, A. Medvedev, Eur. Phys. J. Special Topics 224(8), 1519 (2015)

37. P. Jaros, P. Perlikowski, T. Kapitaniak, Eur. Phys. J. Special Topics 224(8), 1541 (2015)

38. T.E. Vadivasova, V.S. Anishchenko, et al., Eur. Phys. J. Special Topics 224(8), 1553 (2015)

39. P. Saha, D.C. Saha, A. Ray, A.R. Chowdhury, Eur. Phys. J. Special Topics 224(8), 1563 (2015)

40. S. Vaidyanathan, V.-T. Pham, C.K. Volos, Eur. Phys. J. Special Topics 224(8), 1575 (2015)

41. Y. Feng, J. Pu, Z. Wei, Eur. Phys. J. Special Topics 224(8), 1593 (2015)

42. P. Jaros, L. Borkowski, B. Witkowski, K. Czolczynski, T. Kapitaniak, Eur. Phys. J. Special Topics 224(8), 1605 (2015)

43. Y. Feng, Z. Wei, Eur. Phys. J. Special Topics 224(8), 1619 (2015)

44. M. Shahzad, V.-T. Pham, A. Ayad Ahmad, S. Jafari, F. Hadaeghi, Eur. Phys. J. Special Topics 224(8), 1637 (2015) 